\title{
Realidad aumentada en la enseñanza
}

\author{
Rosangela Zaragoza Pérez y Ana Leticia Cuevas Escudero
}

\begin{abstract}
Resumen
El uso de los dispositivos móviles entre la comunidad estudiantil del Colegio de Ciencias y Humanidades ( $\mathrm{cH}$ ), plantel Sur, (en todo momento) puede ser utilizado como una ventaja en ámbitos educativos y de aprendizaje, con la ayuda de las herramientas tecnológicas adecuadas para ello. En este artículo se presenta CCHAR "RA Instrumento de enseñanza en Taller de Cómputo", aplicación basada en realidad aumentada (RA), la cual se compone de 14 modelos 3D, de diferentes dispositivos que contiene la computadora, con el objetivo de ser usada como herramienta didáctica en los aprendizajes del programa de estudio de la materia Taller de Cómputo.
\end{abstract}

Palabras clave: realidad aumentada, dispositivos móviles, Taller de Cómputo.

\section{AUGMENTED REALITY IN TEACHING}

\begin{abstract}
The use of mobile devices among the student community of the College of Science and Humanities (ccH), South campus, (at all times) can be used as an advantage in educational and learning environments with the appropriate technological tools for it. This article presents CCHAR "RA Computer Workshop Teaching Instrument", an application based on augmented reality (RA), which consists of 14 3D models of different devices contained in the computer, with the aim of being used as a teaching tool in the learning of the Computer Workshop curriculum.
\end{abstract}

Keywords: augmented reality, mobile devices, Computer Workshop.

Recepción: 25/02/2020. Aprobación: 01/09/2020.

Dol: http://doi.org/10.22201/cuaieed.16076079e.2020.21.6.9 
"Realidad aumentada en la enseñanza"

\section{Rosangela Zaragoza Perez}

rousszp@hotmail.com orcid.org/0000-0002-9928-5178

Maestra en Ingeniería Ambiental por la Universidad Nacional Autónoma de México (UNAM) e Ingeniería en computación por el Instituto Politécnico Nacional (IPN). He trabajado por 8 años en la docencia en el Colegio de ciencias y Humanidades impartiendo las asignaturas de Taller de cómputo y matemáticas I- II. Cuento con varios diplomados en docencia y Tecnología en la Información y Comunicación tic para la enseñanza. He impartido cursos y talleres en tecnologías para los profesores y en tutoría para los alumnos en el ccr plantel Sur. He desarrollado una aplicación con uso de Realidad Aumentada como apoyo en la enseñanza de la asignatura de Taller de Computó, coautora del libro Plataformas Libres para la Educación Medida por las TIC.

\section{Ana Leticia Cuevas Escudero}

ecla07@hotmail.com

Maestra en Docencia en Biología, Licenciada en Educación Media en el Área de Ciencias Naturales, Ana Leticia Cuevas Escudero, con 17 años de experiencia docente en nivel medio superior, catedrática de biología, con mas 7 diplomados, 80 cursos, 15 cursos impartidos, 20 ponencias y 15 conferencias impartidas, así como charlas de divulgación científica en congresos en diversos eventos académicos, asesora y tutora de alumnos vulnerables, coordinadora y organizadora de eventos académicos institucionales, 1 articulo publicado, coordinadora de grupos de trabajo, fui Secretaria Académica del Consejo Académico del Área de Ciencias y Experimentales, eh sido jurado calificador en eventos de la UNAM. Eh participado como ponente y organizadora de congresos, simposios y trabajo de campo, actualmente colaboro como docente de biología I a IV en ENCCH.Sur. unAM.

\section{Introducción}

La integración paulatina de las TIC (Tecnologías de la Información y la Comunicación) y las TAC (Tecnologías del Aprendizaje y la Comunicación), en el proceso de enseñanza-aprendizaje, se ha traducido en el replanteamiento de conceptos, prácticas y comportamientos, tanto de los profesores como del alumnado. Este cambio de paradigma permite la creación de aplicaciones que involucren la Realidad Aumentada (RA) como una tecnología emergente.

La RA puede incorporar información digital (videos, imágenes, URL) y física en tiempo real. Dicha información es soportada por dispositivos móviles o computadoras personales (Cabero y García, 2016), las cuales cuentan con una cámara para leer los marcadores, disparadores $O$ QR, según el nivel de implementación de realidad aumentada.

Por lo anterior, los niveles de RA se dividen en cuatro. Los activadores QR de nivel cero (0) contienen enlaces hacia materiales como videos, imágenes, documentos, etcétera. Los marcadores nivel uno (1) son figuras geométricas (de blanco y negro), que al ser tomados con la cámara superponen un objeto en el entorno real. Hay imágenes que funcionan como activadoras (disparadores) de nivel dos (2), las cuales tendrán el objeto en 3D o un GPS. Por último, la visión aumentada hace uso de gafas o lentes biónicos, de nivel tres (3). 
"Realidad aumentada en la enseñanza"

Gráfica 1. Los resultados de la evaluación inicial muestran en promedio tres respuestas correctas por alumno los que indica un bajo conocimiento de los temas relacionados a la asignatura. Donde el resultado de las preguntas relacionadas al hardware está cercana al promedio de respuestas correctas dada por el alumnado, presentado bajo \% de alumnos que aciertan correctamente respecto al tema mencionado anteriormente.
La realidad aumentada con fines educativos presenta una didáctica diferente en la reproducción y creación de materiales, que están apoyados de un dispositivo móvil (smartphone), aparato de uso común entre los jóvenes, para potencializar las capacidades de los sentidos. Es decir, se aborda la experiencia del uso de las TIC y TAC, y los conocimientos e interacción con el entorno educativo del alumno, como una manera de potencializar los aprendizajes.

\section{Desarrollo}

Si consideramos que los alumnos utilizan de manera cotidiana dichos dispositivos móviles, es sorprendente identificar, en la asignatura de Taller de Cómputo, ciertas dificultades conceptuales en las tecnologías, principalmente en el aprendizaje "Describe el hardware de acuerdo con su funcionalidad y características", ubicado en la Unidad II. Hardware y software. De un total de 241 alumnos atendidos en el ciclo escolar 2019, 34\% identifican correctamente el concepto de hardware respecto al software y 30\% identifican los diferentes dispositivos con los que cuenta el hardware de una computadora (ver gráfica 1).
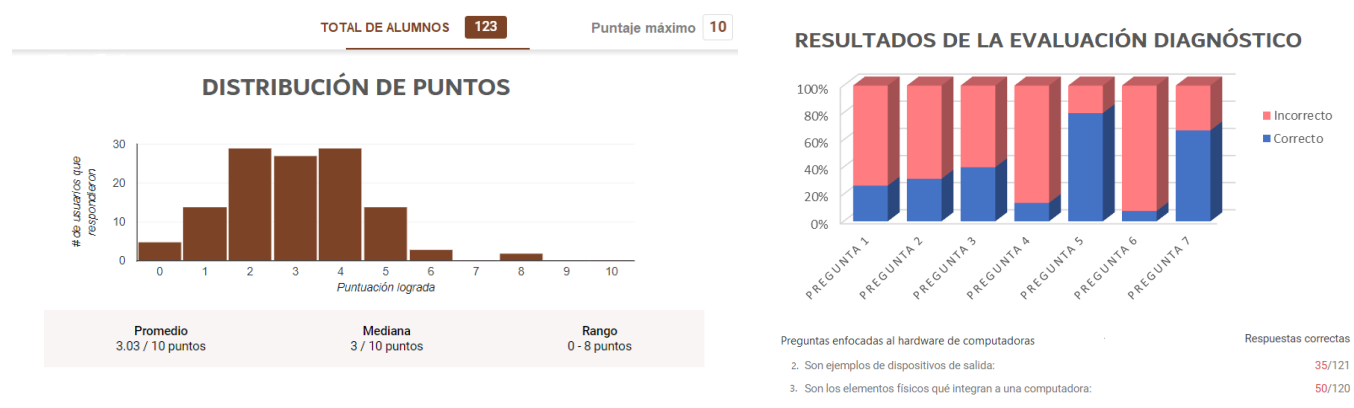

Por ello se creó, para la asignatura de Taller de Cómputo, una aplicación móvil, que involucrara la herramienta habitual entre los jóvenes (celular), para trasladarlo a un ambiente educativo pero atractivo para ellos. La combinación de los elementos anteriores y de la realidad aumentada -puesto que enriquece la información y facilita la comprensión, dado que se pueden observar objetos desde cualquier punto- crea simuladores y laboratorios seguros para los estudiantes (Cabrero-Almenara, 2018), además de ser disruptiva. Así, se tiene como resultado la aplicación CCHAR, pensada para dispositivos móviles (Tablet y Smartphones), así como para las dos plataformas más populares (Android e ios).

Por tanto, para la selección de los componentes se contempló el aprendizaje y temática del programa de estudio se la asignatura Taller de Cómputo: los dispositivos y sus características más representativos de las microcomputadoras. Debido a ello, el modelado de las imágenes 3D se realizó en el software Maya y SketckUp, considerando un total de 14 componentes representativos para el diseño y desarrollo de la aplicación (ver imagen 1). 
Imagen 1. Componentes de la computadora.

Imagen 2. Elementos que componen la realidad aumentada.

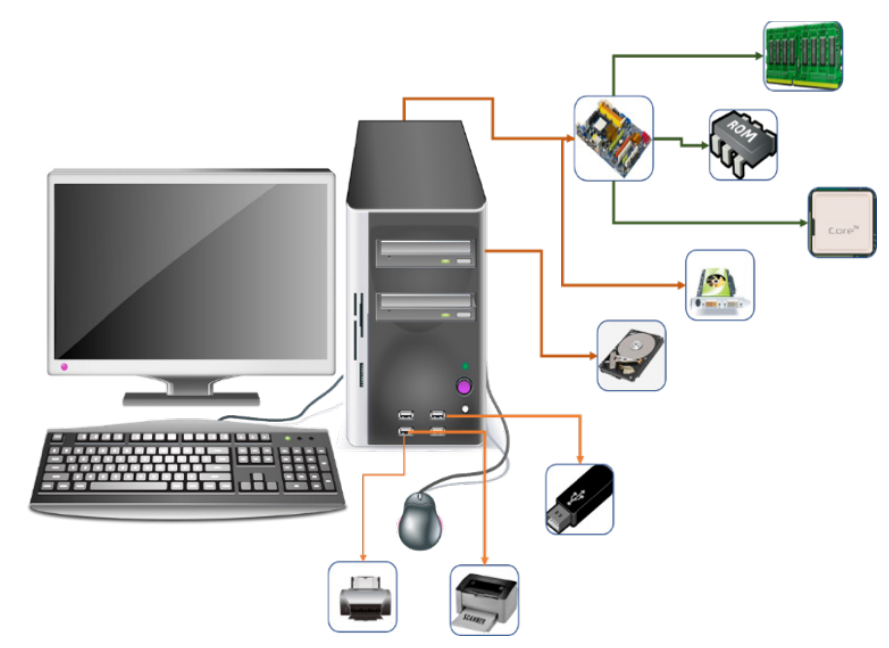

En la segunda fase de implementación de la RA, se requirió que la Pc o el dispositivo contara con cámara, un procesador -que se encarga del mayor trabajo en la plataforma de RA-, software de desarrollo (Vuforia y Unity), una pantalla para visualizar el objeto y, por último el disparador, (target = imagen). La imagen 2 muestra el proceso general de implementación de realidad aumentada.

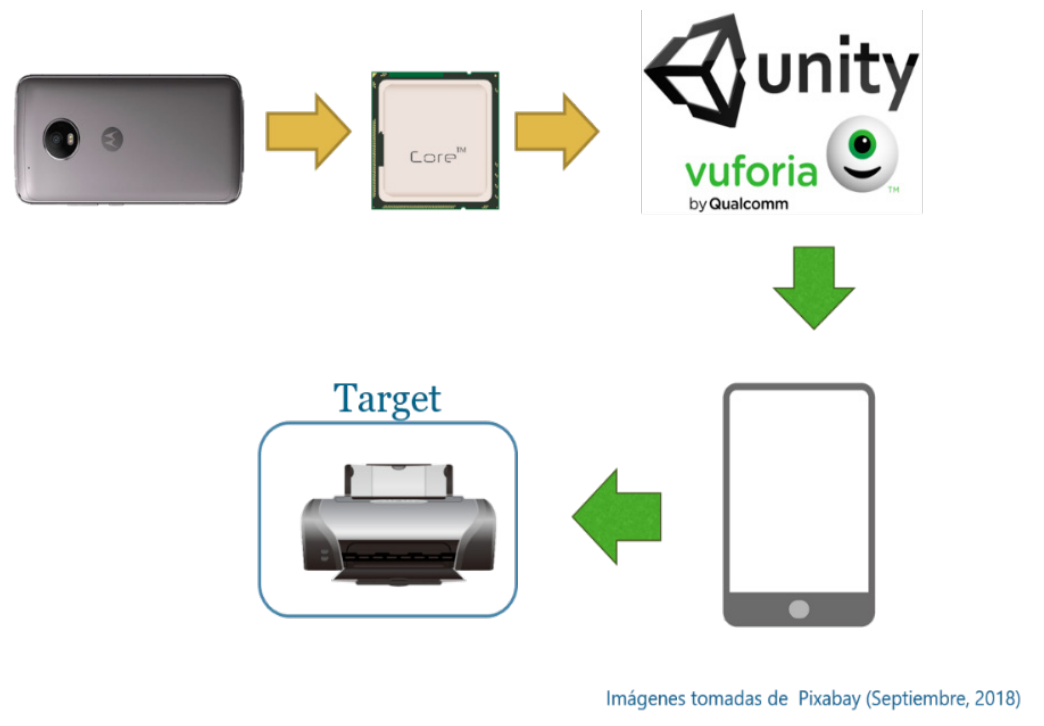

La imagen 3 muestra el entorno de trabajo, empleado en el proyecto PB102019, para la construcción de la aplicación: es necesario un motor central (Vuforia), que es el kit de desarrollo de software (SDK) de RA empleado para generar los APK (Android Aplication Package), las llaves y la base de datos de los disparadores (imágenes). La plataforma de desarrollo de Unity, software de videojuegos en 3D y ambientes de RA, contiene rutinas de programación que permiten el diseño, creación y funcionamiento de entornos interactivos, con el cual se construyeron los componentes del hardware de la computadora. La combinación del motor central y la plataforma de desarrollo (Vuforia + Unity) dan como resultado la CCHAR (logo representativo de la aplicación), material enfocado a la asignatura de 
Imagen 3. Entorno de desarrollo
Taller de Cómputo de una manera interactiva que interpone objetos en 3D, para ser ampliados en un espacio real.

Lo anterior permite a los alumnos conocer los componentes de la computadora, siendo ellos quienes controlen el proceso de aprendizaje, al tomar la decisión de cuando aumentar la información y combinar lo real con lo virtual (Fombona et al., 2012).

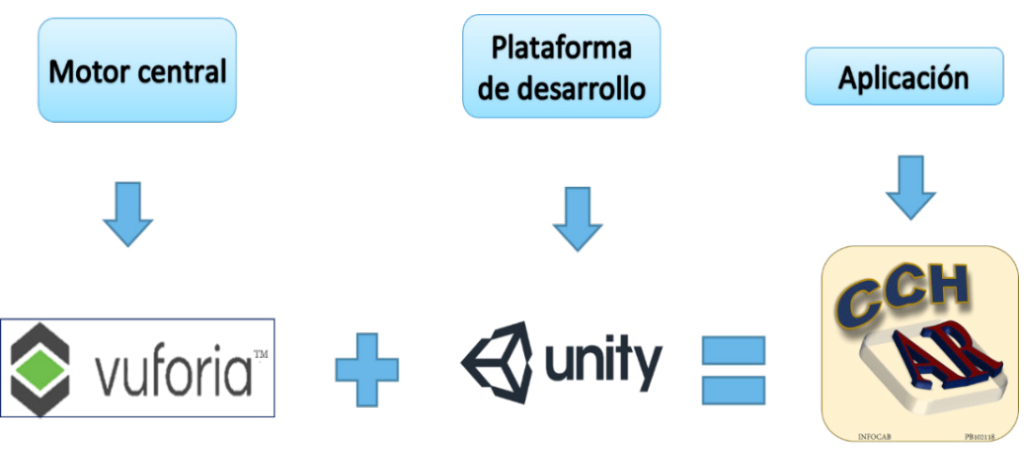

\section{Consideraciones finales}

En la conferencia "Realidad aumentada: herramienta innovadora para la educación", dirigida a alumnos, se presentaron los avances de la aplicación. El objetivs eran que el alumno, en dispositivos Android (Samsung J7) con el APK instalado, interactuara con la herramienta, y se conociera la opinión de los jóvenes. Las primeras observaciones realizadas a la aplicación fueron que la cama no detectaba bien la imagen, que no era posible interactuar con el objeto en el dispositivo, y que se perdía la información rápidamente. La imagen 4 muestra las primeras versiones de la aplicación.
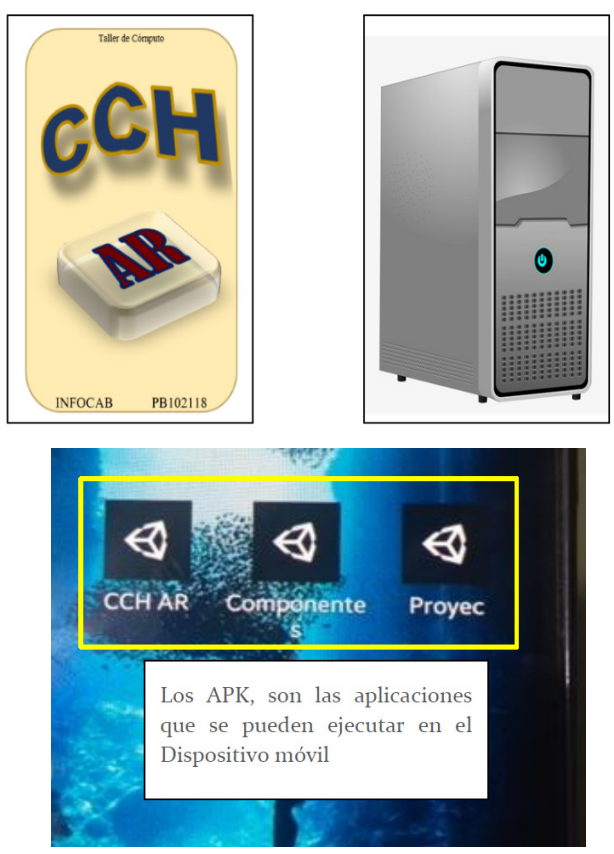
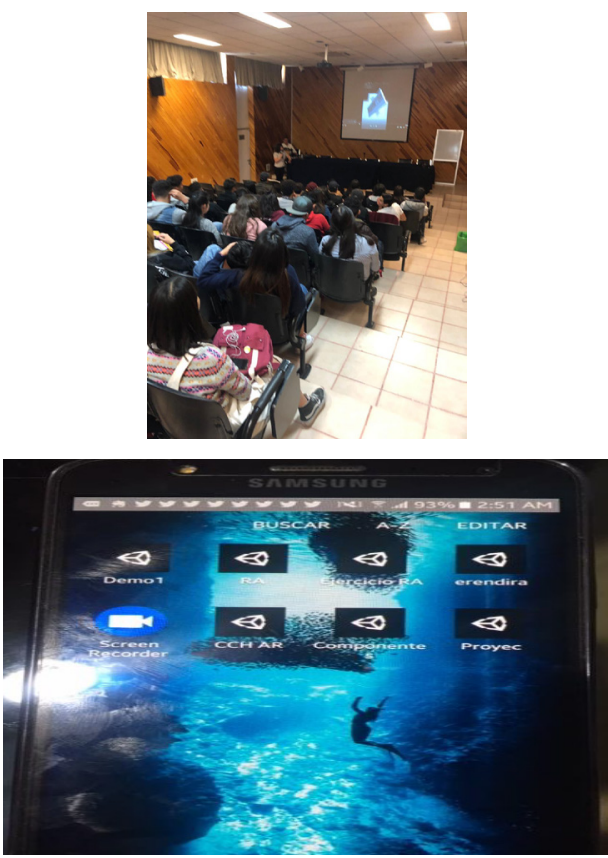

Imagen 4. En la parte superior se muestran las primeras versiones de la aplicación, en la parte inferior izquierda se muestran un ejemplo de los disparadores diseñados para ser leídos por el ApK, a la derecha una imagen de la presentación de la versión beta de CCHAR. 
Imagen 5. Disparadores. QR de la aplicación CCHAR.

Imagen 6. Entorno de CCHAR.

Lado izquierdo, tienda de

Google Play. Lado superior derecho, instrucciones de uso. Parte inferior derecha, muestra el target y su entorno (RA).
Finalmente, la aplicación se presentó en la Muestra Integral de Materiales Didácticas con Apoyo de tic y los Servicios Audiovisuales, donde también los alumnos y profesores pudieron interactuar con la aplicación final, es decir, descargarla de las tiendas correspondientes de Apple y/o Android bajo el nombre de "RA Instrumento de Enseñanza" en Taller de Cómputo. Al ser ejecutada, la aplicación $(a p p)$ cuenta con un pequeño manual de uso. Además, se incluye una breve descripción de cada componente. Los disparadores fueron proporcionados por los integrantes del equipo; es importante mencionar que éstos sólo pueden ser integrados como imagen en la propia aplicación y que se integrara un link con los disparadores en la siguiente actualización (ver imagen 5). La imagen 6 muestra la aplicación en funcionamiento.

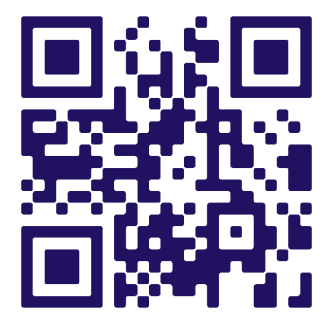

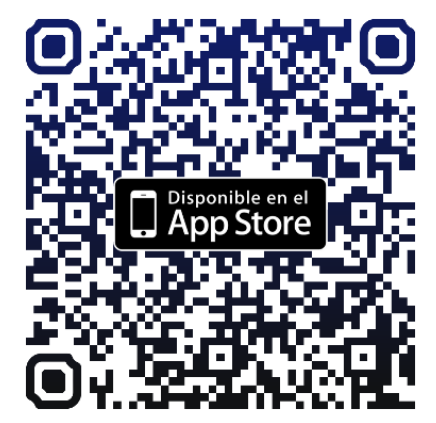
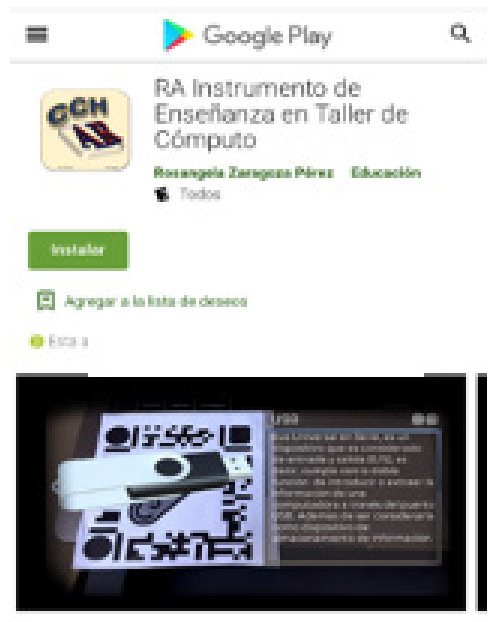

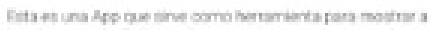

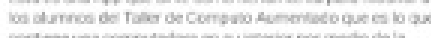

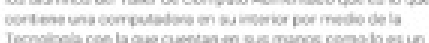
smerthore.

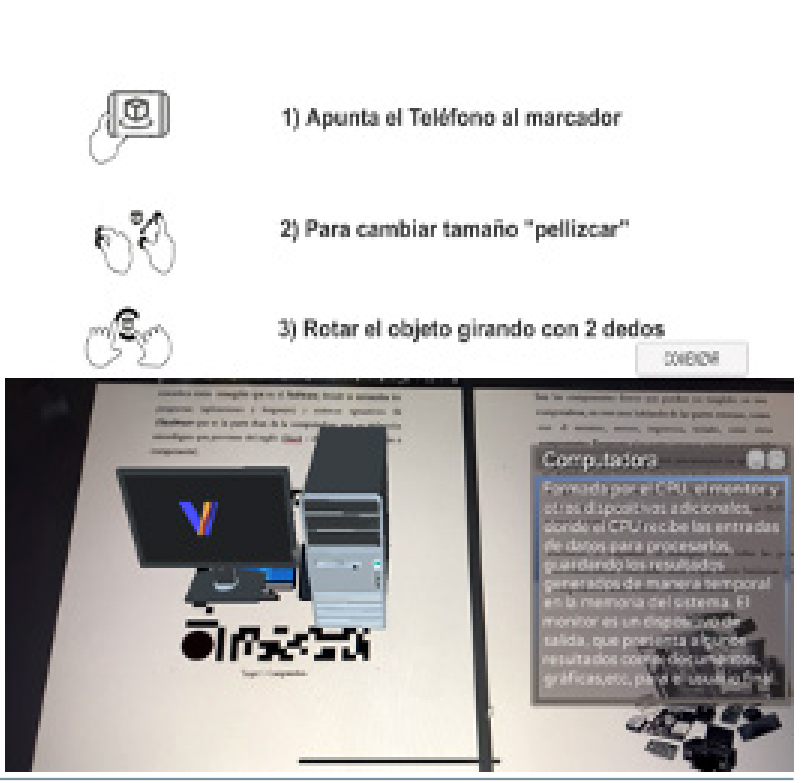




\section{Conclusiones}

La realidad aumentada es uno de esos avances que, con un buen uso, se puede aprovechar para mejorar la didáctica y hacer materiales creativos e innovadores, motivando el aprendizaje significativo y colaborativo de los estudiantes.

Por esa razón, la aplicación RA Instrumento de enseñanza es un importante material de apoyo para reforzar el aprendizaje en la materia de Taller de Cómputo, que emplea la Ra en el diseño de contenido creativo, para motivar el trabajo en el aula, donde el alumno podrá seguir un proceso de autoaprendizaje.

Por el momento, la aplicación está disponible en la tienda de Android o ios de manera gratuita para la comunidad docente, que la podrá incluir en sus actividades de forma libre. El trabajo siguiente es implementar el material en el aula como estrategia de aprendizaje, puesto que la aplicación fue liberada a finales del ciclo 2020-1. Entre los proyectos para esta app están publicar los materiales en la RUA (Red Universitaria de Aprendizaje) y portal académico para su rápido acceso, subir una nueva versión de la aplicación para ser usada en un libro de actividades (se encuentra en proceso de edición), e integrar y modelar algunos componentes adicionales.

\section{Referencias}

* Redacción BBc Mundo. (2016, 17 octubre). Qué es la realidad aumentada, cómo se diferencia de la virtual y por qué Apple apuesta fuertemente a ella. BBC News. https://www.bbc.com/mundo/noticias-37678017

* Cabero-Almenara, J., de la Horra Villacé, I. y Sánchez Bolado, J. (2018). La realidad Aumentada como herramienta educativa. Aplicación a la educación Infantil, Primaria, Secundaria y Bachillerato. Paraninfo.

* Fombona Cadavieco, J., Pascual Sevillano, M. A., Ferreira Amador, M. F. M. (2012, julio). Realidad aumentada, una evolución de las aplicaciones de los dispositivos móviles. Píxel-Bit. Revista de Medios y Educción, 41, 197-210. https://www.redalyc. org/articulo.oa?id=36828247015

* Gómez Rodríguez, D. M. (2017, julio-diciembre). La innovación educativa basada en la tecnología. Revista Multi-Ensayos, 2(4), 102-106. https://revistasnicaragua.net. ni/index.php/multiensayos/article/view/2976

* de la Horra Villacé, I. (2016). Realidad aumentada, una revolución educativa. EDMETIC, 6(1), 9-22. Dol: https://doi.org/10.21071/edmetic.v6i1.5762

* Posada Prieto, F. (2014). Realidad Aumentada en el aula. Canaltic. http://canaltic. $\mathrm{com} / \mathrm{blog} / \mathrm{p}=1859$

* Villalustres, L. y del Moral, M. E. (2016). Experiencias interactivas con realidad aumentada en las aulas. Octaedro. 


\section{Cómo CITAR ESTE ARTículo}

* Zaragoza Pérez, Rosangela y Cuevas Escudero, Ana Leticia. (2020, noviembrediciembre). Realidad aumentada en la enseñanza. Revista Digital Universitaria (RDU), 21(6). Dol: http://doi.org/10.22201/cuaieed.16076079e.2020.21.6.9 